\title{
Biochemical Pharmacology Research of Rhubarb in Traditional Chinese Medicine
}

\author{
Yanfei Sun ${ }^{1, a}$ \\ ${ }^{1}$ Aba Teachers University, Aba, Sichuan, China, 623002
}

Keywords: Biochemical Pharmacology Research, Rhubarb, Traditional Chinese Medicine

\begin{abstract}
In recent years, the domestic pharmacological research and clinical application of rhubarb are far more breakthroughs the scope of the role of diarrhea can be explained, it is widely used in internal and external women and children and other clinical diseases of acute and chronic diseases. Its medicinal methods and dosage forms are also varied, now we study the Chinese medicine rhubarb biochemical pharmacology.
\end{abstract}

\section{Introduction}

Rhubarb is one of the commonly used drugs. In recent years, China's research on rhubarb has made new breakthroughs and found that rhubarb and compound rhubarb can effectively treat acute illness, supernatant tract bleeding, hyperlipidemia, bacterial and viral infections, hepatitis, renal insufficiency, vasculitis, Burns, pharmacological studies have shown that rhubarb has diarrhea, inhibition of small intestine smooth muscle, regulate the stomach and pancreas, enhance immune function, but also has to promote bile secretion, bleeding, cooling, kidney protection and anti-tumor effect. The excellent function of rhubarb is increasingly concerned by scholars both at home and abroad. The clinical and pharmacological studies in this area are summarized.

\section{The Chemical Composition of Rhubarb}

Modern medicine research rhubarb rhizome contains a variety of bioactive ingredients, the inorganic components of at least 25 kinds of elements. Namely, anthracene derivatives, stilbene compounds, tannins, organic acids, volatile oils and so on. Anthracene derivatives are divided into: free anthraquinone derivatives such as aloeemodin, chrysaron, chrysophanol, emodin, isoernodin, D (laccaic acid D), emodin (physcion), rhein (rhein); combined with anthraquinone compounds, rhein, aloe emodin, chrysophanol mono- and bisglucoside, emodin, emodin Anthranins and anthrone compounds, rhein, palmidin and glycosides such as sennoside A, B, C, D, E, F and so on. Stilbene compounds such as rhaponticin, 3,5,4'-trihydroxystin-4'-O- $\beta$-D- (6'-O-gallogic acid) glucoside (3,5,4 (6'-O-gallayl) -glucoside), 3,5,4'-trihydroxystyrene-4'-O- $\beta$-D-glucopyranoside naphthalene derivatives such as torachrysone-8-O- $\beta$-D-glucopy ranoside, torachrysone-8- (6 \& apos; -D-glucopyranoside, -oxaly) -glucoside and torac-hryson. Tannins: gallocao dextrose, d-catechins, gallic acid, rhodopsin (tetrann) and so on. Rhubarb tetraphenylene by hydrolysis was gallic acid, cinnamic acid and rhubarb Ming (rheosmin). In addition to containing resin, still contains organic acids: malic acid, succinic acid, oxalic acid, lactic acid, cinnamic acid, isobutylene acid, citric acid, Rhubarb also contains volatile oil, fatty acids and phytosterols. Recently, from the rhubarb in the diarrhea under the diarrhea elements rhein-osides A, B and C, D, identified, A and B for a pair of epimers, the structure of 8-0- $\beta$-D C-D-glucopyranosyl-9-anthrone; $\mathrm{C}$ and $\mathrm{D}$ are another epimeric, 8-O- $\beta$-D-glucosyl 10-C- $\beta$-D-glucose-based rhein-9-anthrone. Rhubarb contains a variety of inorganic elements: potassium $(\mathrm{K})$, calcium $(\mathrm{Ca})$, magnesium $(\mathrm{Mg})$, lanthanum $(\mathrm{La})$, copper $(\mathrm{Cu})$, zinc $(\mathrm{Zn})$, manganese $(\mathrm{Mn})$, iron $(\mathrm{Fe})(\mathrm{Pb})$, drilling $(\mathrm{Co})$, nickel $(\mathrm{Ni})$, titanium $(\mathrm{Ti})$, platinum $(\mathrm{Pt})$, mercury (Hg), germanium (Ge), high iron, manganese and zinc content, , Nickel content is low. 


\section{The Impact on the Digestive System}

Diarrhea Effect. The active ingredient of rhubarb is mainly sour supplements after oral administration, in the digestive tract by the metabolism of bacteria as a biological activity of metabolic products and play diarrhea role. Sectin in the intestinal bacteria under the action of decomposition of rhein anthraquinone, rhein anthraquinone can stimulate the large intestine mucosa, the increase in peristalsis and diarrhea. In addition can inhibit the intestinal cell membrane $\mathrm{Na}+, \mathrm{K}$ + -ATP enzyme, impede $\mathrm{Na}+$ transport, so that intestinal osmotic pressure, to retain a lot of water, promote peristalsis and diarrhea. Another study has proved: rhubarb play a role in diarrhea is another way to diarrhea glycosides absorbed by the small intestine, the liver into aglycones, and then stimulate the gastric plexus plexus caused by large intestinal peristalsis diarrhea, while part of the prototype or aglycone Blood transported to the large intestine, stimulate the submucosal plexus and deeper muscle plexus, etc., so that intestinal hyperactivity, causing diarrhea. Rhubarb diarrhea ingredients can be excreted in the milk, milk after taking can affect the baby, causing infant diarrhea. Rhubarb has the dual effect of excitement and inhibition of the gastrointestinal tract. The material basis of the former is sennoside, and the material basis of the latter is tannin. Experiments show that: Dahuang Tang on the early gastrointestinal tract of mice was hyperactivity and late exercise was inhibited, low concentration, high concentration inhibition. Rhubarb contained in the tannin on gastrointestinal motility has inhibitory effect, so in the production of diarrhea may occur after constipation. Large doses of rhubarb $(1 \sim 5 \mathrm{~g})$ resulted in diarrhea effect; small doses of rhubarb (0.05 0.3g) when there constipation, the mechanism and rhubarb contained in the convergence of tannin cover the content of too little diarrhea the role of the next component.

Gallbladder. Paul liver rhubarb is the medicine of dampness and red medicine in the "Compendium of Materia Medica" that is the treatment of jaundice records. In modern medical research, emodin and rhein can promote bilirubin and bile acid secretion, increase bilirubin and bile acid content, chemical poisoning caused by animal liver injury and liver damage animal morphology changes are protected or eliminated, Significantly reduce the SGPT value, restore the normal function of liver cells; promote liver cell regeneration and liver cell RNA synthesis; stimulate the human body to produce interferon; increase gallbladder contraction, relaxation Odyssey's sphincter, intrahepatic capillary bile duct is conducive to the elimination of inflammation, Promote liver cell regeneration, row of stone. Clinical also shows that: rhubarb can reduce the jaundice index and SGPT value, restore liver function, and can prevent the transformation of mild hepatitis to severe hepatitis, reduce complications and reduce mortality.

Promote Pancreatic Secretion. Inhibition of trypsin activity rhubarb can promote the secretion and excretion of pancreatic juice, so that pancreatic juice flow increased. On trypsin, pancreatic elastase, pancreatic protease, pancreatic kallikrein, pancreatic lipase and amylase activity has a significant inhibitory effect, but also inhibit the digestive action of pepsin, but does not affect the secretion of gastric acid secretion of gastrin The The active ingredients of this effect are water-soluble, non-tannin and anthraquinone glycosides. Therefore, rhubarb is a clinical treatment of acute severe pancreatitis commonly used drugs. In addition, Chinese medicine that rhubarb bitter cold Shangwei, may be related to the role of digestive enzymes.

\section{The Impact on the Blood System}

Hemostatic Effect. Rhubarb bitter cold settlement, commonly used in the treatment of blood heat caused by the vomiting hematemesis, Nvxue, more in the compound application, the use of single flavor rhubarb can also be. It is characterized by the exact effect of hemostasis, quick. The hemostatic active ingredient is $\alpha$-catechins, gallic acid. Hemostatic mechanism is: to promote platelet adhesion and aggregation function, is conducive to thrombosis; increased platelet count and fibrinogen content, shortening the clotting time; reduce the activity of thrombin III, promote blood clotting; The In addition, gallic acid can increase a2-macroglobulin (a2-MG) content, reduce fibrinolytic activity, accelerate blood clotting. 
Hypolipidemic. (TC), triglyceride (TG), low density lipoprotein (LDL), very low density lipoprotein (VLDL) and lipid peroxidation were significantly lower in serum and liver, and high density lipoprotein / TG The ratio increases. May be due to diarrhea effect of rhubarb and affect the absorption of cholesterol.

Clinically proven rhubarb can affect hemorheology, so that blood viscosity dropped to normal, lower cholesterol, triglycerides. But also blood pressure, animal experiments show that rhubarb has to promote the role of blood into the liver, while reducing the decomposition of glycogen. Yueshi made with rhubarb capsules, treatment of hyperlipidemia clinical efficacy is obvious. Chen to rhubarb powder-based treatment of high blood viscosity syndrome, the general medication for a month, more than three months of blood viscosity can be reduced to normal. But also increase plasma osmolality. Magic on the one hand through the permeability of diuretic blood pressure, on the other hand can make the peripheral tissue fluid flow to the blood vessels, play the role of internal infusion, to remove the microcirculation after bleeding is beneficial, and have local vasoconstriction Permeability to reduce the hemostatic effect. Therefore, clinical for acute and chronic stroke, regardless of hemorrhagic or ischemic can play a role in improving microcirculation, antihypertensive, fever, prevention of the role of infection, help to reduce and prevent the occurrence of sequelae. Rhubarb Tongluo cathartic catharsis diarrhea, diarrhea fire and blood, blood to stop bleeding, can lead the stool, excreted evil heat and dirty things, adjust the function of organs, thereby accelerating the recovery of stroke patients and clinical symptoms of improvement, shorten the acute phase Treatment. Chen Shi with rhubarb dipping liquid treatment of stroke, compared with the treatment with dehydrating agent is not only effective, and no electrolyte imbalance risk.

\section{The Anti-Infective Effect}

Anti-Pathogenic Microorganisms. Rhubarb antibacterial spectrum of wide, sensitive bacteria is staphylococci, hemolytic streptococcus, gonorrhea, diphtheria bacilli, Salmonella typhi, dysentery bacilli and so on. Sensitive viruses have influenza virus, orphans virus, hepatitis B virus, poliovirus and so on. Other sensitive microbes are amoeba protozoa, vaginal trichomoniasis, schistosomiasis and leptospirosis. The antibacterial active ingredient is rhein, emodin, aloe emodin. Antimicrobial mechanism is an enzyme system that affects folic acid; inhibits bacterial nucleic acid and protein synthesis; inhibits bacterial biooxidase systems; induces interferon.

Anti-Inflammatory, Antipyretic Effect. Studies have shown that rhubarb can reduce the body temperature of infected patients and animals, rabbits in the cooling at the same time, the third ventricle perfusate PGE and CAMP levels were significantly reduced, reducing the central heat medium. In addition, rhubarb can prepare erythrocyte membrane $\mathrm{Na}+, \mathrm{K}+-$ enzyme active agent oxidative phosphorylation process, reduce the production and consumption of ATP, reduce heat production, energy metabolism at a low level, but also conducive to lower body temperature.

Treatment of High Fever. Practice has proved that rhubarb has anti-infection and antipyretic cooling effect. Rhubarb can restore the permeability of the intestinal mucosa and capillaries and monophage cell phagocytosis, effectively avoid the absorption of intestinal endotoxin, so that cloudy foreign phase, evil is complex, yin and yang to balance. Lin with rhubarb (alone or with the use of Wu) treatment of high fever, the effect is very good, that is, no side effects, also found no recurrence. He made a single flavor rhubarb made of new Qingning tablets, the treatment of acute suppurative tonsillitis, antipyretic cooling fast, and no rebound phenomenon. Rhubarb is also made into powder, preparation, topical treatment of mumps, suppurative otitis media and so on.

\section{Conclusion}

The medicinal resources of rhubarb are very rich, and it is widely used in the world as a world-accepted natural medicine. It has anti-tumor, antihypertensive, anti-aging, antifungal, antipyretic and analgesic effects. To rhubarb as raw materials to develop a variety of different effects of the new formulations still has a huge development space. Clinical uses are detoxification, cooling blood to stop bleeding, gallbladder withdrawal yellow and blood circulation. Therefore, this 
article further study these plants found that the drug has the development and utilization of the value of the physiological active ingredients. However, due to the study of pharmacology has just started, therefore, the focus of the study is to strengthen the toxic research.

\section{References}

[1] Liu Shenggui, Peng Siwen, Yidan. The inhibition role of the 18 kinds of anti-inflammatory Chinese herbal medicine[J]. Modern Chinese Medicine, 2007, 27 (2): 41 - 43.

[2] Zhou Xiong, Xuan Lijiang Chinese medicine sheep hoof chemical composition and pharmacological effects of research [J]. Zhejiang Journal of Traditional Chinese Medicine, 2006, 41 (3): 180-182.

[3] Huang Qing, Qu Xing, Wu Qing. Huang Lian, earth rhubarb, Atractylodes extract anti-psoriasis experimental study [J]. Chinese Journal of Traditional Chinese Medicine, 2008, 15 (6): 30-31. 